\title{
PERCEPÇÕES DE CONSTRUTORES E INCORPORADORES SOBRE INDUSTRIALIZAÇÃO NA CONSTRUÇÃO CIVIL EM CURITIBA
}

\section{RESUMO}

Ainda que reconhecido pelo seu potencial para impulsionar a expansão da indústria da construção civil, a pré-fabricação representa uma pequena parcela do mercado brasileiro atual. $\mathrm{O}$ presente artigo foi elaborado com o objetivo de analisar o nível de conhecimento e aceitação de construtores e incorporadores sobre o emprego de processos construtivos industrializados para edificações na cidade de Curitiba/PR, contribuindo para a tomada de decisão entre processos tradicionais e industrializados. Por meio de um levantamento com profissionais da região, foi possível traçar um perfil das empresas participantes, bem como suas experiências prévias com industrialização. Também foram analisadas as percepções quanto às principais barreiras e benefícios ao utilizar processos industrializados. Os benefícios que apresentaram maior relação com a pré-fabricação foram atribuídos às características relacionadas ao tempo, à qualidade, aos impactos ambientais e à gestão do canteiro, sendo a redução de tempo e o aumento de produtividade os maiores motivadores para empresas. Para barreiras, a falta de conhecimento/ experiência e os maiores prazos de planejamento foram os itens com maior concordância quanto à relação com a industrialização. Contudo, as barreiras que mais restringem o seu uso foram: a falta de especificação de pré-fabricação em projeto e o aumento de custo. Por fim, sugere-se a continuidade deste artigo com a expansão do escopo para todo o território nacional.

Palavras-Chave: Industrialização. Construção Civil. Benefícios. Barreiras. Motivadores. 


\section{INTRODUÇÃO}

O setor da construção civil no Brasil é de grande expressividade para a geração de riquezas para o país. São 6,4\% de participação no valor adicionado bruto - a preços básicos - no ano de 2015 (CBIC, 2016), ou seja, 325 bilhões de reais em riquezas produzidas para o setor.Além disso, o déficit habitacional de aproximadamente seis milhões de moradias impulsiona o crescimento constante do setor (FUNDAÇÃO JOÃO PINHEIRO, 2015).

Contudo, pesquisas no setor da construção civil assinalam diversos pontos que necessitam de aprimoramentos. Koskela (2000) faz um apanhado dos problemas enfrentados por este setor durante o Século XX nos países nórdicos, Estados Unidos da América e Reino Unido. Altos custos, baixo crescimento da produtividade e baixa qualidade são os problemas mais frequentes levantados e podem ser atribuídos recorrentemente à má gestão e à falta de organização.

A baixa produtividade do setor de construção civil foi estudada por Hewage e Ruwanpua (2006) no Canadá. Os estudiosos verificaram que carpinteiros passavam apenas metade das horas de trabalho efetivamente realizando atividades produtivas. No Brasil, um estudo elaborado pelo Sinduscon (2015) concluiu que, apesar do aquecimento setorial entre os anos de 2003 e 2013, a produtividade manteve-se praticamente estática e cerca de 30\% inferior à média da economia.

Muito se fala na literatura nacional einternacional sobre a contribuição positiva da industrialização em situações de obra. Dentre as vantagens exploradas, tem-se a redução de cronograma, o acréscimo de qualidade, a utilização de mão de obra especializada, a redução de impactos ambientais, maior valor agregado, entre outros (GOODIER; GIBB, 2005).

Sob o ponto de vista ambiental, estudos de caso recentes verificam a redução do impacto ao optar pela construção fora do canteiro. Dentre os benefícios que a industrialização pode trazer, estão: (1) Redução de resíduos na construção; (2) Redução de materiais utilizados no empreendimento (água, madeira, aço etc.); (3) Redução da energia incorporada e emissões (CAO et al., 2015; MONAHAN; POWELL, 2010; MAO et al.,2013; WANG; TAM,2015; JAILLON; POON, 2013; AYE et al., 2012; LI et al., 2014).

Ainda que diversos benefícios da construção industrializada tenham sido explorados na literatura, a sua adoção continua limitada a uma pequena parcela das empresas que atuam no mercado de edificações na construção civil. A Pesquisa Anual da Indústria da Construção do ano de 2013 mostra que, naquele ano, em obras e/ou serviços de construção de edificações no Brasil, o percentual de participação em serviços de montagem de edifícios pré-fabricados foi inferior a $1 \%$ do valor total das incorporações.

Com este estudo, pretende-se contribuir para o entendimento e a ampliação do mercado da construção industrializada no Brasil, especificamente para a cidade de Curitiba, através da divulgação das percepções de construtores e incorporadores da capital paranaense quanto aos seus principais benefícios/motivadores e desvantagens/barreiras. Objetiva-se analisar o nível de conhecimento e aceitação para esses processos, contribuindo para a tomada de decisão para a escolha dos sistemas construtivos de uma obra. 


\section{DESENVOLVIMENTO}

\subsection{Revisão da literatura}

A expansão da indústria da construção civil brasileira depende, em grande parte, dos processos construtivos industrializados. Sua difusão poderá contribuir para a mitigação do acentuado déficit habitacional brasileiro e ainda permitir a redução de custos e prazos, assegurando a garantia e rapidez de entrega. Contudo, para que se possa alcançar a construção industrializada em larga escala, diversos desafios deverão ser vencidos (MONTEIRO FILHA et al., 2010). Os autores dividem esses desafios em quatro grupos:

- Tecnologia-A industrialização em massa implica padronização de produtos e processos. Contudo, a inserção em grande escala exige mudanças em normas técnicas, reorganização da cadeia produtiva e qualificação da mão de obra. Outros desafios identificados são as significativas diferenças entre códigos de obras municipais, além da dificuldade para se estabelecer critérios para homologação, avaliação e aprovação de processos construtivos inovadores.

- Financiamentos - $\mathrm{O}$ setor da construção civil brasileira possui baixa relação entre a soma dos financiamentos imobiliários e o PIB, ainda que este setor seja muito dependente de crédito. Uma solução proposta pelos autores é a definição de uma política de financiamento que utilize o crédito como um incentivador, fomentando o interesse por inovação nos diferentes elos da cadeia produtiva.

- Mercado-Um dos grandes desafios nesta categoria é a eliminação do déficit habitacional no Brasil, principalmente para as classes de baixa renda, pois concentram pouco mais de $90 \%$ desta demanda. Ainda que este quadro represente um potencial de crescimento para a construção industrializada, seu emprego requer alinhamento de toda a cadeia produtiva e padronização de materiais de construção.

- Concorrência - As empresas que atuam no setor da construção civil dependem da capacidade de obtenção de crédito para aumentar sua competitividade, sendo o barateamento do custo do capital um fator essencial. Além disto, a concorrência é influenciada pelos preços, pela qualidade, pela sustentabilidade ambiental e pelas reduções no tempo de obra.

O relatório do Estudo Prospectivo Setorial da construção civil brasileira (CGEE, 2009) apresenta a "[...] visão do futuro do segmento de construção habitacional industrializada para 2024". Foram propostas macroações relevantes para as seis rotas estratégicas consideradas, que foram estruturadas em forma de diretrizes para os anos de 2009 a 2024. O relatório elenca três fatores chave de alavancagem para alcançar a visão:(1) Soluções inovadoras; (2) Mão de obra qualificada e certificada; (3) Materiais, componentes e sistemas construtivos industrializados em conformidade com as normas técnicas.

\subsubsection{Percepções de Stakeholders sobre industrialização da construção civil}

A fim de reestruturar o processo de tomada de decisão sob uma perspectiva dos Stakeholders, levantamentos com construtores, engenheiros, clientes, arquitetos e uma ampla gama 
de envolvidos passaram a ser realizados em diferentes países. Esses estudos têm impulsionado a elaboração de modelos de apoio à tomada de decisão durante a fase de planejamento de empreendimentos quanto ao uso de processos construtivos industrializados. Dois exemplos de ferramentas fundamentadas em percepções de Stakeholders são a pioneira IMMPREST (Interactive Methodfor Measuring PRE-assembly and STandardisation benefit in construction), desenvolvida na Universidade de Loughborough, no Reino Unido (BLISMAS et al., 2003), e a PPMOF (Prefabrication, Preassembly, Modularization, and Off-site Fabrication) decision framework, desenvolvida pelo CII (Construction Industry Institute), nos Estados Unidos da América (CII, 2002).

O início do processo de elaboração dessas ferramentas foi descrito por Pendlebury e Gibb (2004). Segundo os autores, após a publicação do relatório subsidiado pelo governo britânico, Rethinking Construction (EGAN, 1998), a Construction Industry Research and Information Association (CIRIA) percebeu que seria necessário realizar pesquisas para compreender quais os potenciais benefícios, bem como as formas correntes de emprego da "padronização e pré-montagem" (standardisation and pre-assembly, $\mathrm{S} \& \mathrm{P})$ na indústria da construção civil. Em consequência, parcerias foram estabelecidas e diversos relatórios foram publicados.

Um dos frutos destas parcerias foi o Standardisation and Pre-Assembly - Client's Guide and Toolkit (GIBB, 2000), que é um guia prático e conjunto de ferramentas que contém uma lista de ações, opções e procedimentos amparados por informações de suporte, que visam auxiliar as tomadas de decisão relacionadas a processos construtivos industrializados.
Posteriormente, houve refinamento deste guia ao agregar informações de um estudo, elaborado por Gibb e Isack (2003), sobre as necessidades que motivavam clientes durante a tomada de decisão (PENDLEBURY; GIBB, 2004).

Gibb e Isack (2003) entrevistaram cinquenta e nove funcionários de alto escalão, representando quarenta e duas das maiores e mais frequentes organizações de clientes do Reino Unido. Foram levantados os benefícios e as desvantagens da pré-fabricação (preassembly). Os benefícios citados com maior frequência foram, do mais frequente para o menos: tempo, qualidade,custo, produtividade, segurança. Quanto às desvantagens, as principais relacionadas foram: operacional (limitações da edificação ou instalação em questão), qualidade, custo, disponibilidade, projeto.

Durante Workshops para complementação da ferramenta que estava sendo desenvolvida pela Universidade de Loughborough e a CIRIA, constatou-se que os profissionais participantes estavam familiarizados com os benefícios da construção industrializada. Além disso, notou-se que, ao identificar os aspectos desvantajosos e alinhá-los com estes benefícios, uma nova estratégia passava a ser construída. A partir de uma lista com quatorze motivadores e vinte restrições, procedeu-se com um levantamento enviado a um grupo heterogêneo de profissionais da construção civil. Foram 289 (duzentos e oitenta e nove) questionários enviados, com uma taxa de retorno de 25,3\%. Os autores separaram as respostas em quatro grupos de restrições, a saber: processo, valor,cadeia produtiva e conhecimento. Por fim, foram propostas ações para mitigação de cada uma das restrições encontradas (BLISMAS et al., 2005). 
Uma nova pesquisa, dessa vez com foco nas oportunidades para o mercado de pré-fabricados, foi publicada por Goodier e Gibb (2007). Mais de oitenta questionários foram completados, tendo como foco três grupos de Stakeholders: fornecedores/montadores, construtores, projetistas/clientes. Constatou-se que as possibilidades e o potencial da industrialização eram de conhecimento dos respondentes. Também se percebeu que a maior parte compreendia as vantagens e desvantagens de seu uso. Quanto à análise feita sobre o potencial do mercado de industrialização na construção, foram levantados dois problemas que devem ser enfrentados para que este mercado se desenvolva:
- falta de informações transparentes para a tomada de decisão, principalmente relacionadas às comparações de custo com métodos tradicionais;

- falta de mão de obra qualificada. Em primeira análise, foi julgada como uma oportunidade para a construção industrializada. A falta de trabalhadores qualificados também se mostrou uma barreira para os fornecedores.

O IMMPREST Toolkitv.1.3 traz uma lista de motivadores e restrições muito similar àqueles apresentados pelos estudos descritos nesta seção. $\mathrm{O}$ quadro a seguir apresenta uma versão traduzida destes fatores.

Quadro 1: Motivadores e Restrições para o uso de industrialização na construção civil

\begin{tabular}{|c|c|c|}
\hline & & MOTIVADORES \\
\hline \multirow{4}{*}{ D.1 Custo } & 1.1 & Garantir a segurança do custo do projeto \\
\hline & 1.2 & $\begin{array}{l}\text { Minimizar custos não construtivos (e.g. Gerenciamento, projeto, } \\
\text { orçamento, etc) }\end{array}$ \\
\hline & 1.3 & Minimizar custos construtivos \\
\hline & 1.4 & $\begin{array}{l}\text { Minimizar os custos globais do ciclo de vida (e.g. manutenção } \\
\text { mais eficiente) }\end{array}$ \\
\hline \multirow{3}{*}{ D.2 Tempo } & 2.1 & Garantir a segurança da data de conclusão \\
\hline & 2.2 & Minimizar duração em canteiro \\
\hline & 2.3 & Minimizar tempo global do projeto \\
\hline \multirow{3}{*}{ D.3 Qualidade } & 3.1 & Conseguir alta qualidade (e.g. acabamentos, tolerâncias) \\
\hline & 3.2 & Conseguir previsibilidade da qualidade \\
\hline & 3.3 & $\begin{array}{l}\text { Conseguir previsibilidade do desempenho ao longo do ciclo de } \\
\text { vida de uma instalação }\end{array}$ \\
\hline D.4 Saúde e Segurança & 4.1 & Reduzir os riscos de saúde e segurança \\
\hline \multirow{3}{*}{ D.5 Sustentabilidade } & 5.1 & $\begin{array}{l}\text { Reduzir impactos ambientais durante a construção (e.g. reduzir } \\
\text { desperdícios) }\end{array}$ \\
\hline & 5.2 & Maximizar o desempenho ambiental ao longo do ciclo de vida \\
\hline & 5.3 & Implementar respeito aos princípios das pessoas \\
\hline
\end{tabular}




\begin{tabular}{|c|c|c|}
\hline \multicolumn{3}{|r|}{ RESTRIÇÕES } \\
\hline \multirow{7}{*}{ C. 1} & 1.1 & $\begin{array}{l}\text { Leiaute ou espaço restrito em canteiro (e.g. local para } \\
\text { armazenamento) }\end{array}$ \\
\hline & 1.2 & Interfaces comerciais múltiplas em áreas de trabalho restritas \\
\hline & 1.3 & Mão de obra qualificada em canteiro é restrita ou muito cara \\
\hline & 1.4 & $\begin{array}{l}\text { Um problema transportar produtos manufaturados para o } \\
\text { canteiro }\end{array}$ \\
\hline & 1.5 & Ambiente de trabalho ativo limita as operações em canteiro \\
\hline & 1.6 & Limitação em movimentar elementos pré-fabricados pelo canteiro \\
\hline & 1.7 & $\begin{array}{l}\text { Restrições em canteiro por entidades externas (e.g. vizinhos, } \\
\text { restrição de ruídos) }\end{array}$ \\
\hline \multirow{5}{*}{ C.2 Processo } & 2.1 & $\begin{array}{l}\text { Projeto com curta duração total (e.g. pouco tempo para projetos } \\
\text { ou testes) }\end{array}$ \\
\hline & 2.2 & $\begin{array}{l}\text { Impossibilidade de fixar o projeto em estágios iniciais para se } \\
\text { adequar à industrialização }\end{array}$ \\
\hline & 2.3 & Decisões chave já fazem exclusões à industrialização \\
\hline & 2.4 & $\begin{array}{l}\text { Não é possível usar os mesmos processos / procedimentos em } \\
\text { projetos subsequentes }\end{array}$ \\
\hline & 2.5 & $\begin{array}{l}\text { Não há oportunidade para repetividade do componente neste } \\
\text { ou em projetos futuros }\end{array}$ \\
\hline \multirow{8}{*}{ C.3 Aquisição } & 3.1 & $\begin{array}{l}\text { Membros da equipe de projeto não possuem experiência prévia } \\
\text { com industrialização }\end{array}$ \\
\hline & 3.2 & Obrigação em trabalhar com uma única cadeia de fornecimento \\
\hline & 3.3 & Não dispostos a se comprometer com um único fornecedor \\
\hline & 3.4 & Obrigação em aceitar menor custo em vez de melhor valor \\
\hline & 3.5 & Capacidade limitada de fornecedores \\
\hline & 3.6 & Expertise limitada em inspeções fora do canteiro \\
\hline & 3.7 & $\begin{array}{l}\text { Inexistência de orientações e conselhos durante estágios iniciais } \\
\text { da construção / manufatura }\end{array}$ \\
\hline & 3.8 & Obrigação em aceirar um custo específico \\
\hline
\end{tabular}

Fonte: adaptado de Blismas et al. (2003)

Pesquisas descritivas sobre percepções de profissionais envolvidos com as práticas de industrialização na construção são recentes, mas têm se mostrado relevantes para a evolução deste segmento. Desde então, diversos estudos com esta abordagem foram publicados em países como China, Estados Unidos, Austrália e Reino Unido (TAM et al., 2007; LU; LISKA, 2008; BLISMAS; WAKEFIELD, 2009; AZHAR etal.,2013; O'CONNOR etal.2014). Contudo, não foram encontrados pelo autor levantamentos realizados com profissionais brasileiros. 


\subsubsection{Conceitos e terminologia}

Para esta pesquisa, é importante que sejam esclarecidos alguns termos e conceitos pertinentes. Segundo a NBR 9062 (ABNT, 2006):

\section{“3.10 elemento pré-moldado: \\ Elemento moldado previamente e fora do local de utilização definitiva na estrutura, conforme especificações estabelecidas em 12.1.1. \\ 3.11 elemento pré-fabricado: \\ Elemento pré-moldado executado industrialmente, em instalações permanentes de empresa destinada para este fim, que se enquadram e atendem aos requisitos mínimos das especificações do item 12.1.2."}

Dentre os requisitos citados nos itens 12.1.2 desta norma, encontra-se: a mão de obra, que deve ser treinada e especializada; matéria-prima qualificada com estrutura específica para controle de qualidade e inspeção do processo produtivo; elementos produzidos com auxílio de máquinas e equipamentos industriais que racionalizem e qualifiquem o processo.

$\mathrm{Na}$ literatura internacional, há diversos termos para industrialização na construção civil. A diferença de nomenclatura pode ser em função de, por exemplo: regionalismo, época de criação, abrangência. Entretanto, todos os termos apresentados a seguir se relacionam com a execução de processos construtivos fora do canteiro (GOODIER; GIBB, 2005; CIDB, 2010; RAHMAN, 2014; WRAP, 2007; PAN et al., 2007):
- Off-site Production (OSP - Produção fora do canteiro);

- Off-site Fabrication (OSF-Fabricação fora do canteiro);

- Off-siteManufacturing(OSM-Manufatura fora do canteiro);

- Off-site Construction (OSC - Construção fora do canteiro);

- Preassembly (Pré-montagem);

- Prefabrication (Pré-fabricação);

- Industrialized Building Systems (IBS Sistemas Construtivos Industrializados);

- Modern Methods of Construction (MMC Métodos Modernos de Construção).

Os processos construtivos industrializados podem ser divididos em quatro categorias:

- Nível 1 - Manufatura de componente e submontagem

- Nível 2 - Pré-montagem não volumétrica

- Nível 3-Construção modular

- Nível 4-Pré-montagem volumétrica

Estas categorias estão descritas e exemplificadas na Figura 1 (GIBB; ISACK, 2003). 
Figura 1: Categorias de industrialização na construção civil

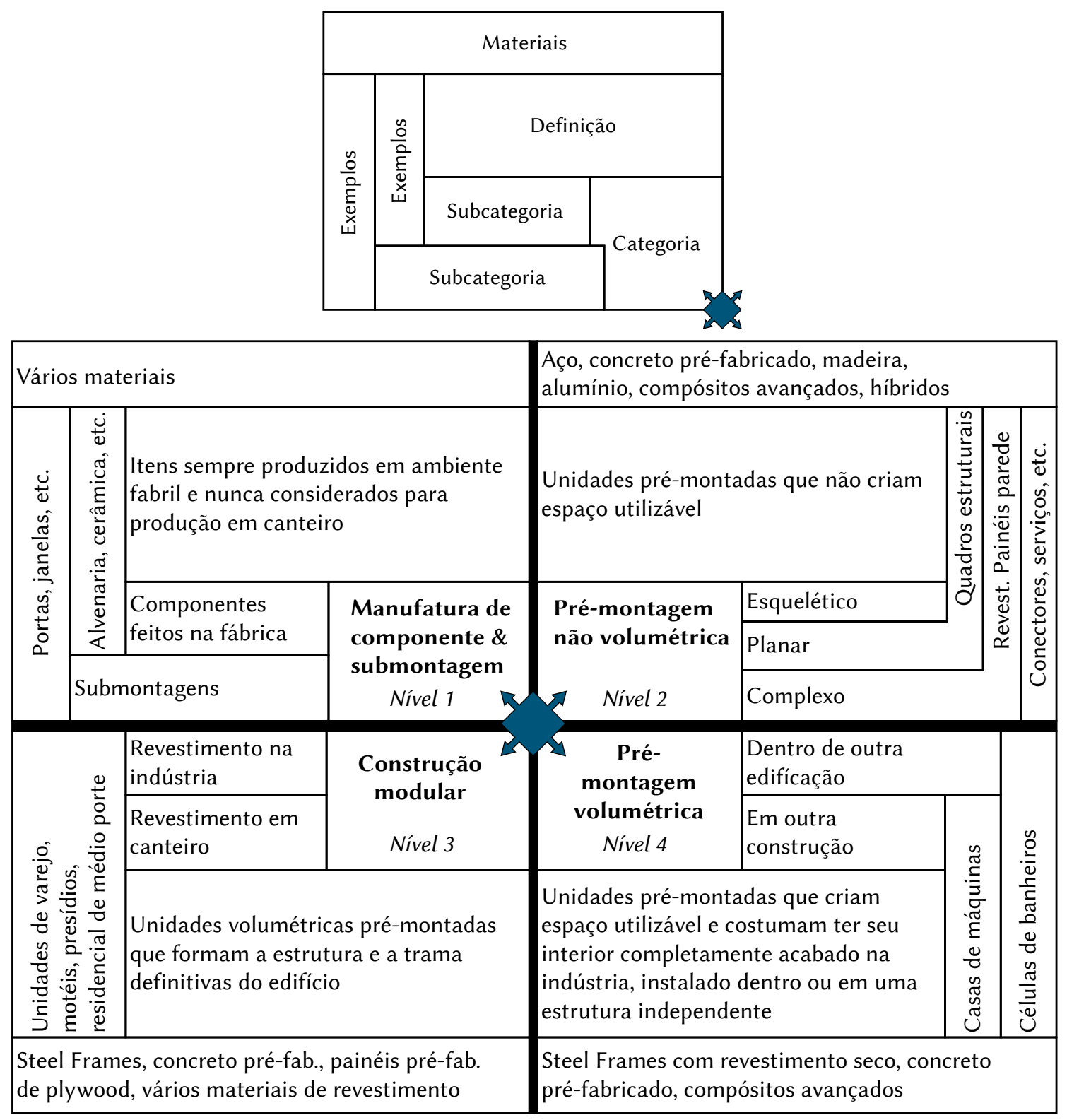

Fonte: Adaptado de Gibb e Isack (2003)

As próximas subseções abordam os procedimentos metodológicos do levantamento das percepções discutidas anteriormente dos construtores e incorporadores de empresas situadas em Curitiba/PR.

\subsection{Procedimentos}

\section{Metodológicos}

Em vista da problemática proposta, esta pesquisa terá natureza aplicada. A abordagem do problema será quantitativa, pois as informações geradas serão traduzidas em números, analisadas e classificadas com auxílio de recursos e técnicas estatísticas. Quanto aos objetivos, a pesquisa será descritiva e assumirá forma de levantamento (GIL, 2002).

Babbie (2003) define dois desenhos básicos para pesquisas de levantamento, também tratadas pelo termo inglês survey, a saber: Surveys interseccionais; Surveys longitudinais. Nesta pesquisa, será seguido o desenho dos levantamentos 
interseccionais, em que os dados são colhidos em um determinado momento, a fim "descrever alguma população maior na mesma ocasião".

O levantamento será realizado de maneira análoga aos estudos apresentados em 2.1. A população de amostra será convidada a responder um questionário sobre visões e experiências com industrialização na construção civil, sendo esta dividida em quatro tipos, conforme descrito no Item 2.1.2. Este artigo trará um compilado de informações, geradas a partir dos retornos recebidos sobre as vantagens/benefícios e as desvantagens/barreiras para utilização da pré-fabricação.

\subsection{População e amostra}

A pesquisa se apoia no princípio básico da abordagem probabilística, que afirma que uma amostra será representativa de uma população se todos os membros tiverem oportunidades iguais de serem selecionados para a amostra. Amostras estas, denominadas como MIPSE - método de igual probabilidade de seleção(BABBIE,2003).

Portanto, arbitrou-se que a amostra seria composta por empresários, engenheiros ou arquitetos de empresas cujo CNPJ estivesse classificado segundo o item "4.1 - Construção de Edifícios” da Classificação Nacional de Atividades Econômicas (CNAE 2.0), além de que deveriam possuir cinco ou mais funcionários e sede em Curitiba/PR.A seleção foi resultado de uma busca no Cadastro das Indústrias do Paraná 2015, da FIEP.

Cento e noventa e nove foram os resultados encontrados. Em complemento, outros dezenove profissionais foram selecionados pelos autores, todos enquadrados nos requisitos previamente descritos. Por conseguinte, a amostra inicial ${ }^{1}$ foi de 218 profissionais.

A amostra considerada representa aproximadamente $40 \%$ da população total de estabelecimentos ativos na construção civil, com 5 ou mais funcionários e enquadrados no grupo de Construção de Edifícios (CNAE 4.1). Segundo dados extraídos do banco de dados do CBIC (2016), concluiu-se, adotando as mesmas proporções do estado do Paraná, que Curitiba possuía uma população de 568 estabelecimentos ativos em 2014, ano da última publicação de dados.

\subsection{Coleta dos dados}

Os questionários enviados foram elaborados com a ferramenta Google Forms, enviados por $e$-mail para toda a população de amostra para serem acessados através de weblink informado no corpo do e-mail. O seu conteúdo tomou como base a dissertação de $\mathrm{Lu}$ (2007), que descreveu, por meio de survey com 131 stakeholders, as percepções quanto à industrialização, além dos principais tipos de industrialização utilizados na indústria estadunidense.

Antes de iniciar a distribuição online da versão definitiva, foram aplicados dois questionários de calibração com duas empresas parceiras, para que fossem identificadas possíveis melhorias. Pequenas alterações foram efetuadas e iniciou-se o envio de $e$-mails.

O questionário é composto por cinco seções, nove questões abertas e nove fechadas. A primeira seção orienta o respondente sobre os quatro tipos de industrialização considerados.

\footnotetext{
${ }^{1}$ Diversos $e$-mails retornaram e tiveram de ser descartados.
} 
A segunda seção consta de oito questões, sendo seis opcionais, com o intuito de entender o posicionamento da empresa no mercado e ante os processos construtivos industrializados.

Por sua vez, a terceira seção explora as opiniões sobre as vantagens e barreiras dos processos industrializados. As percepções elencadas se fundamentaram no estudo de $\mathrm{Lu}$ (2007), bem como no IMMPREST Toolkit v. 1.3 e nos demais artigos citados em 2.1.1. Adaptou-se o método de medição desenvolvido por Rensis Likert, denominado de "escalonamento" Likert, e descrito por Babbie (2003) como um termo associado ao formato da pergunta, que permite que o respondente examine uma determinada afirmação e expresse o seu nível de concordância em diferentes níveis, usualmente: concordam fortemente; concordam; discordam; discordam fortemente.

Por fim, as seções 4 e 5 são uma ramificação da seção que as precede, pois solicitam que sejam apontados os três principais motivos e desafios para que a industrialização seja adotada nas empresas em que os respondentes atuam.

Após envio dos questionários, contatos telefônicos foram estabelecidos para conscientizar os respondentes quanto aos objetivos e à importância que a pesquisa representaria para a indústria regional da construção civil.

\subsection{Análises dos dados}

A relação entre fatores como margem de erro, nível de confiança e tamanho da amostra foi obtida por meio da Equação 1, descrita por Bartlett et al. (2001).

$$
\mathrm{n}=\frac{\mathrm{N} \cdot 2 \cdot \mathrm{p} \cdot(1-\mathrm{p})}{(\mathrm{N}-1) \cdot \mathrm{e}^{2}+\mathrm{Z} 2 \cdot \mathrm{p} \cdot(1-\mathrm{p})}
$$

Onde:

$\mathrm{n}$ - amostra;

$\mathrm{N}$ - população;

Z - variável normal padronizada associada ao nível de confiança;

$\mathrm{p}$ - verdadeira probabilidade do evento;

e - erro amostral.

Os resultados quantitativos obtidos por meio do escalonamento Likert foram interpretados a partir de médias e desvio-padrão amostral. Desta forma, identificaram-se quais itens possuíam maior convergência entre as opiniões levantadas.

\section{RESULTADOS E DISCUSSÃO}

\subsection{Amostragem}

Após envio de $e$-mails e contatos telefônicos, a amostra para envio dos questionários foi de 179 profissionais. Essa redução em relação aos 218 profissionais definidos na subseção 2.3 ocorreu por motivos, como:
- E-mails retornaram, não possuíam websites e/ou telefones estavam inativos para verificação.

- Empresas que estavam registradas no CNAE 4.1, mas não exerciam mais atividades com construções de edifícios.

- Empresas haviam encerrado atividades. 
Definiu-se um nível de confiança de 90\% e, portanto, um Z de 1,645 (OCHOA,2016).Ao todo, foram 27 questionários respondidos (n). Desta forma, a incógnita da Equação 1 passou a ser o erro amostral "e" calculado como 12,37\%.

\subsection{Informações sobre as empresas e uso de técnicas de industrialização}

A idade média dos respondentes foi de 38 anos e os quatro cargos mais comuns foram: engenheiro(a) civil; gerente; sócio(a); diretor.
Quanto ao percentual aproximado do faturamento de 2015, por segmento (Figura 2), incorporação apareceu com o maior percentual (30,3\%). Todas as demais atividades estiveram presentes de forma bem distribuída, com a menor participação para obras industriais $(9,7 \%)$. Todos os respondentes alegaram que a participação da companhia se limitava ao território nacional, sobretudo com atuação exclusivamente regional $(53,8 \%)$, conforme Figura 3.

Figura 2: Percentual aproximado do faturamento de 2015 por segmento

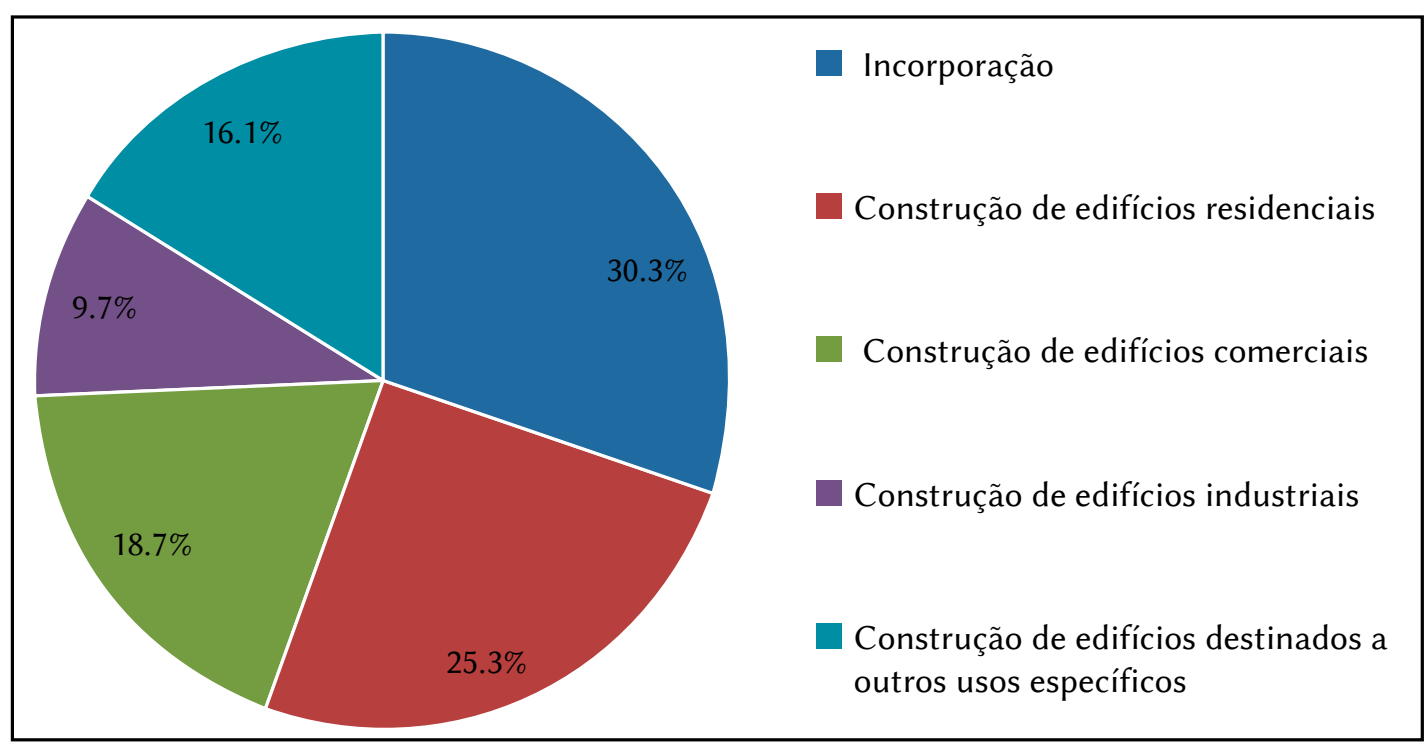

Fonte: Do autor (2016) 
Figura 3: Abrangência da atuação das empresas

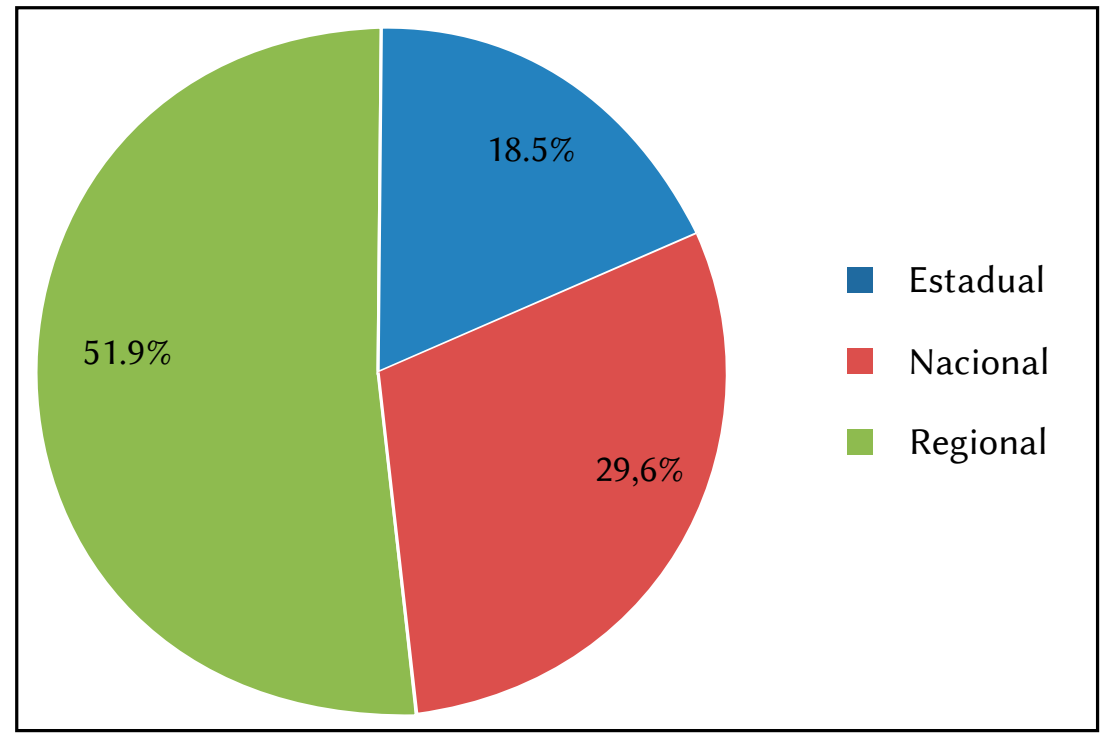

Fonte: Do autor (2016)

Nas sexta e sétima questões, sobre o percentual do faturamento destinado a cada técnica de industrialização descrita, houve grande divergência entre as informações inseridas, além de muitos dos respondentes terem deixado campos sem preenchimento. Por isso, optou-se por desconsiderá-las.

Por fim, avaliou-se o grau de satisfação quanto às experiências passadas com industrialização.
$\mathrm{Na}$ Tabela 1, é apresentado que grande parte dos respondentes já teve experiências com os dois primeiros níveis de industrialização, 88,9 e 70,4\%, respectivamente. Todas as médias ficaram próximas a 4 na escala de 1 a 7 , ou seja, as satisfações médias percebidas são neutras "nem satisfeito, nem insatisfeito". Entretanto, há grande variabilidade de resultados, com desvio-padrão de até 2,6, indicando que as opiniões divergem significativamente.

Tabela 1: Satisfação quanto às experiências passadas com industrialização

Nível de industrialização

1-Submontagens e manufatura de componentes

2-Pré-montagem não volumétrica

3-Pré-montagem volumétrica

4-Construção modular

\section{Média (1-7)}

$\sigma$

Respondentes que utilizaram $\begin{array}{lll}4.4 & 2.1 & 24\end{array}$

$\begin{array}{lll}4.2 & 2.3 & 19\end{array}$

$\begin{array}{lll}4.0 & 2.4 & 7\end{array}$

$\begin{array}{lll}4.2 & 2.6 & 5\end{array}$

Fonte: Do autor (2016) 


\subsection{Percepções sobre a} utilização da industrialização na construção civil

Quando solicitados a avaliar os benefícios da industrialização na construção civil com o escalonamento Likert (1-5), os seis principais indicativos foram: tornam o canteiro mais limpo (4,52); reduzem o impacto ambiental das operações construtivas $(4,44)$; facilitam as medições dos serviços realizados em canteiro $(4,41)$; permitem uniformidade na qualidade dos produtos $(4,41)$; aumentam a produtividade geral do trabalho $(4,37)$; aumentam a eficiência da gestão do canteiro $(4,37)$. Ao todo, quinze dentre os vinte e cinco fatores listados obtiveram médias superiores a 4 . Outros três mantiveram-se abaixo do valor considerado neutro (3), ou seja, não foram vistos como benefícios (Figura 4).

As maiores divergências de percepções ocorreram para os fatores com as menores médias, ou seja, os benefícios considerados mais significativos foram percebidos de maneira mais uniforme pelos profissionais. A redução da necessidade de mão de obra qualificada no canteiro, com média de 2,76, foi a percepção cujas opiniões divergiram de forma mais acentuada, com um desvio-padrão de 1,54. A maior convergência de opiniões ocorreu para a percepção de que reduzem o tempo de execução da obra, cuja média foi de 4,33 e o desvio-padrão de 0,65.

Analogamente, tabularam-se as percepções sobre as barreiras para industrialização na construção civil, representadas na Figura 5. Nessa questão,percebe-se uma maior tendência à neutralidade, ou seja, a maioria das médias se aproxima de 3,0 - não concorda nem discorda. Ademais, também houve maior dispersão dos resultados, sendo o desvio-padrão médio para barreiras $30,1 \%$ superior àquele constatado para os benefícios. 
Figura 4: Vantagens/Benefícios para a utilização das técnicas de pré-fabricação em canteiro

Tornam o canteiro mais limpo

(-) impacto ambiental das operações construtivas

Facilitam as medições dos serviços Uniformidade na qualidade dos produtos

$(+)$ eficiência da gestão de canteiro

$(+)$ produtividade geral do trabalho

(-) tempo de execução da obra

Previsibilidade da qualidade

$(+)$ precisão nos prazos

(-) interferência entre atividades no canteiro

(-) tempo geral de um projeto

Previbilidade do desenpenho

(+) qualidade dos produtos

(+) desempenho ambiental

(-) riscos à saúde e segurança do trabalhador

Custo preciso sem flutuações

(-) espaço para armazenamento em canteiro

Agregam valor ao produto

(-) custos não construtivos

(-) custos construtivos

(-) custo global do empreendimento

(-) custos iniciais do empreendimento

(-) necessidade de MO qualidade no canteiro

Não são afetadas pelas interpéries

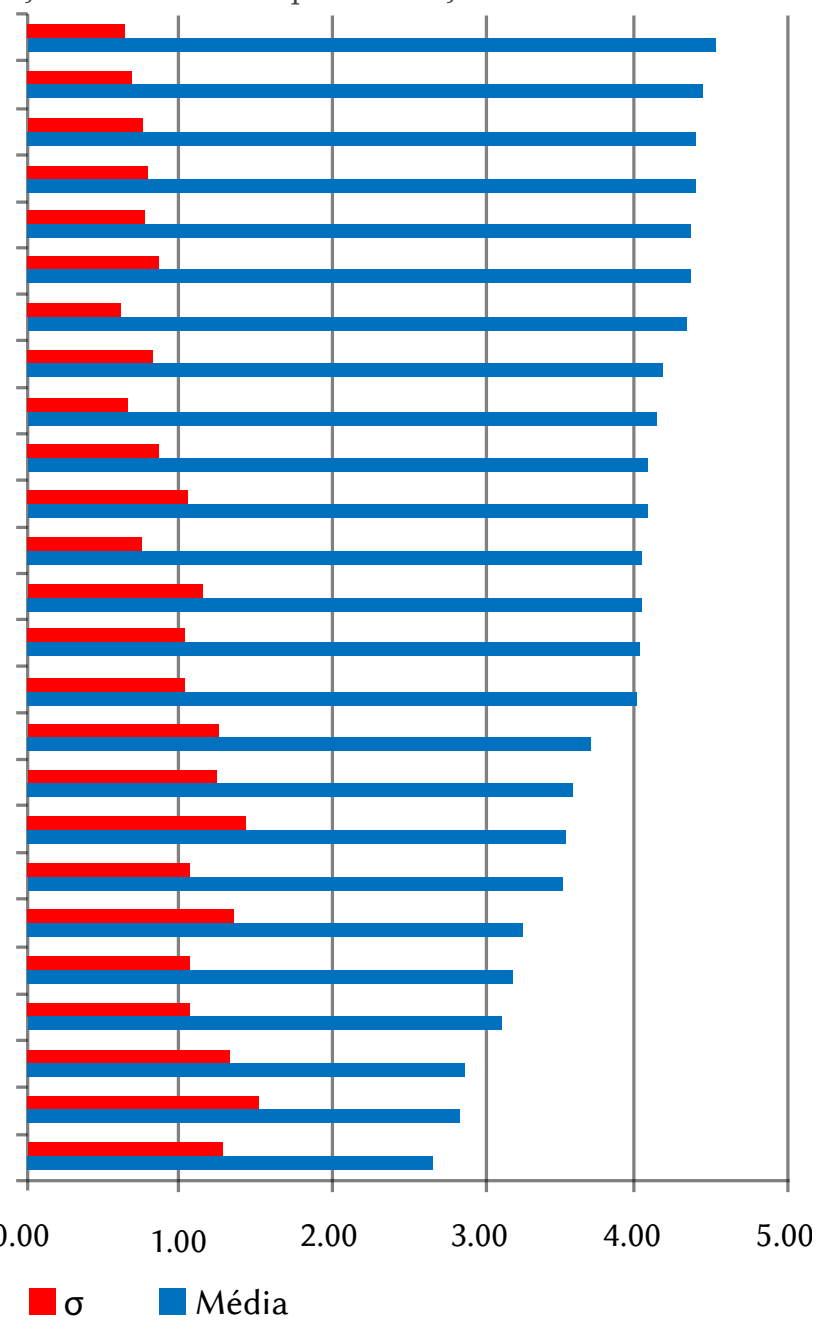

Fonte: Do autor (2016)

A barreira mais significativa foi a falta de conhecimento/experiência que as empresas possuem $(3,67)$, seguida pelos maiores prazos de planejamento necessários para industrializar $(3,63)$, restrições para o transporte dentro do canteiro $(3,56)$, restrições para o transporte atéo canteiro $(3,44)$ e necessidade de um desembolso antecipado de capital $(3,44)$.
As percepções de barreiras criadas pelo curto prazo de execução dos projetos $(3,19)$ e pela visão negativa sobre processos industrializados $(2,67)$ foram as que divergiram de forma mais acentuada, com um desvio-padrão de 1,57. 
Figura 5: Barreiras para a utilização das técnicas de pré-fabricação em canteiro

$(+)$ risco de acidentes de trabalho Há restrições em canteiro por entidades externas Regulamentações locais restringem o uso de pré-fabricação

Visão negativa sobre os processos industrializados Não há repetitividade de porcessos Não é possível repetir em projetos subsequentes Deve-se aceitar um custo específico Não se adequam a projetos pequenos (+) custo global do empreendimento

$\mathrm{O}$ ambiente de trabalho ativo limita as operações em canteiro

Trabalhar com uma única cadeia de fornecimento O projeto sofre constantes nudanças no decorrer da obra

Há maior dificuldade para conseguir financiamentos Deve-se aceitar menor custo em vez de melhor valor Há poucas normas para auxiliar o construtor

Projetos costumam possuir curto prazo de excução

Decisões chave já fazem exclusões à industrialização Não há orietações e conselhos durante estágios iniciais Existem poucos fornecedores Limitam as possibilidades de projeto

Restrições quanto ao leiaute / espaço em canteiro A MO para instalação de elementos pré-fabricados é cara (-) capacidade de realizar mudanças no trabalho em canteiro Não estão dispostos a trabalhar com um único fornecedor Há restrições para o transporte até o canteiro Necessitam de um desenbolso antecipado de capital

Há restrições para o transporte dentro do canteiro Requer maiores prazos de planejamento Empresas possuem pouco conhecimento / Experiência

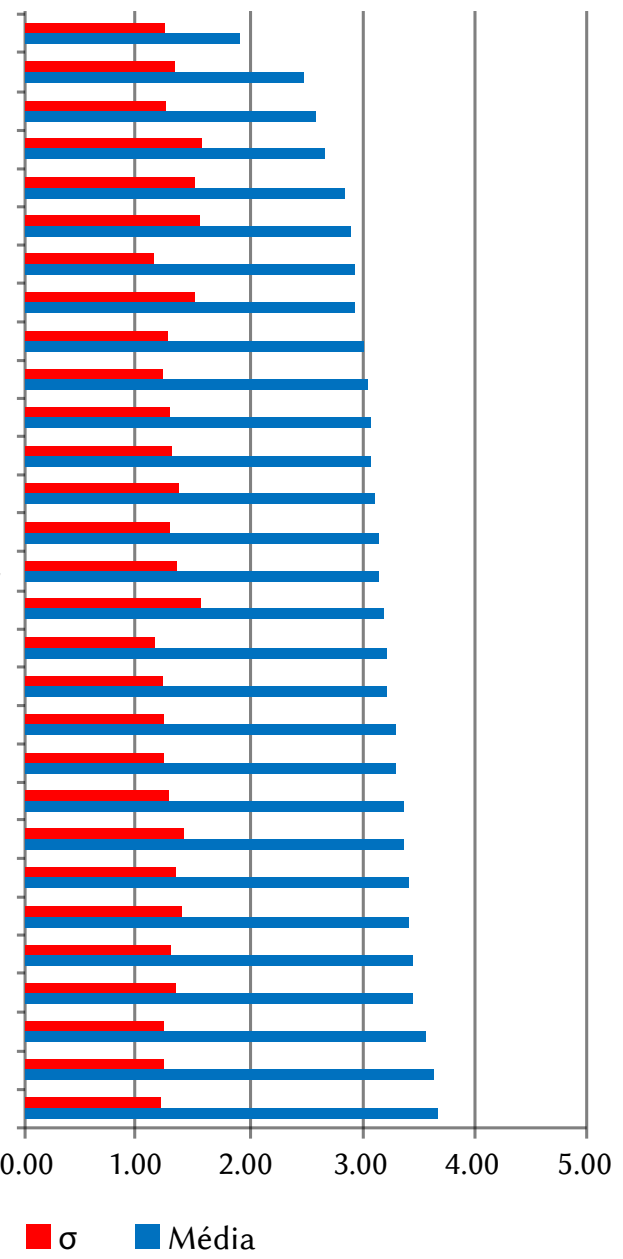

Fonte: Do autor (2016)

A percepção sobre o aumento do custo global do empreendimento, que foi propositalmente inserida em ambas as questões, manteve uma média neutra de 3,00 e 3,11 para barreiras e vantagens (redução do custo), respectivamente.

Outras observações foram acrescentadas pelos respondentes e, de um modo geral, citam o melhor atendimento ao cliente e otimização de projetos, bem como barreiras relacionadas aos paradigmas atuais, à falta de compreensão do impacto financeiro e à corrente dificuldade que os construtores apresentam para planejar seus empreendimentos.
Quando indagados sobre o crescimento do uso de técnicas construtivas industrializadas nos próximos 5 a 10 anos, 89\% dos respondentes afirmaram acreditar que haverá crescimento.As justificativas para tal são diversas e enfatizam os benefícios levantados. Entretanto, a maioria está relacionada às tendências de mercado e à necessidade de adaptação e aperfeiçoamento do setor que, segundo os respondentes, podem ser alcançadas com a industrialização. 
3.4 Motivos para utilizar técnicas de industrialização

\section{na construção}

Nas Tabelas 2 e 3, estão listados os principais motivos pelos quais construtores optam ou deixam de optar por processos industrializados. Cada respondente escolheu três afirmações que julgava exercer maior impacto na tomada de decisão.

Os principais motivadores foram: a redução do tempo de obra (72\%), aumento da produtividade (56\%) e qualidade dos produtos (48\%), além da redução do custo total do empreendimento (36\%). Esses resultados corroboram o levantamento de Gibb e Isack (2003).

Os quatro itens mais citados para desafios e barreiras foram: falta de especificação de pré-fabricados nos projetos (37\%), aumento de custo na construção (33\%), baixa padronização de projetos (33\%), desembolso de capital em estágios iniciais da obra (22\%). Todos os quatro itens se concentram nas dimensões custo e projeto, também levantadas por Gibb e Isack (2003).

Novamente, notam-se pontos de vista completamente opostos sobre os custos de processos industrializados. Ainda que um terço dos respondentes enxergue a industrialização como um potencial de redução de custos, outro um terço acredita que sua adoção tende a encarecer a obra.

Outra dedução plausível é que há maior consenso quanto aos motivadores da industrialização, o que não ocorreu de forma clara para as barreiras, que apresentaram uma distribuição mais heterogênea.

Tabela 2: Motivos para utilizar técnicas de industrialização na construção

Motivos para utilizar técnicas de industrialização na construção
Total de

respostas

18

$72 \%$

Para reduzir o tempo de obra 14 $56 \%$

Para aumentar a produtividade geral do trabalho 12 $48 \%$

Para aumentar a qualidade dos produtos $36 \%$

Para reduzir o custo total do empreendimento 9
Para compensar a falta de mão de obra qualificada 5

Para reduzir retrabalhos 3

Para ter garantia dos custos de execução 3

Para reduzir os impactos ambientais 2

Para aumentar a margem de lucro da empresa

Para melhorar a reputação da empresa

$20 \%$

$12 \%$

$12 \%$

$8 \%$

2 $8 \%$

Para melhorar a gestão do canteiro 


\section{Motivos para utilizar técnicas de \\ industrialização na construção}

Total de

Para compensar as variações climáticas

Para compensar a falta de espaço para armazenamento de materiais em canteiro

Projetistas não especificam pré-fabricados nos projetos

O uso de pré-fabricados aumenta o custo de construção

Pouca repetitividade nos projetos (padronização)

Desembolso inicial de capital

Restrições logísticas

5

Opções limitadas de projeto para pré-fabricados

Pouco conhecimento sobre as técnicas de pré-fabricação

Falta de mão de obra qualificada para montagem dos elementos no canteiro

Instituições financeiras restringem o uso de pré-fabricados

Trabalham com prazos muito curtos para planejamento

$\mathrm{O}(\mathrm{s})$ proprietário(s) não permite(m) o uso de pré-fabricados

Impossibilidade de realizar mudanças no canteiro ao adotar pré-fabricados

Não há ou existem poucos fornecedores locais

3

Não estão dispostos a trabalhar com um único fornecedor

Deve-se aceitar menor custo em vez de melhor valor

2

$7 \%$

Outros: Falta de planejamento e engenharia à montante

Outros: Pouca aceitação do cliente final

1 
Com o objetivo de analisar o nível de conhecimento e aceitação de construtores e incorporadores sobre o emprego de processos construtivos industrializados para edificações na cidade de Curitiba/PR, o presente estudo traça o perfil das empresas envolvidas, bem como as percepções de profissionais sobre vantagens, desvantagens, principais fatores de tomada de decisão e perspectivas para o mercado da construção industrializada.

O perfil dos respondentes é composto principalmente por profissionais da engenharia, com idade média de 38 anos que trabalham em construtoras com atuação nacional, estadual e regional, sendo este o mais recorrente (51,9\%).O faturamento médio destas empresas no ano de 2015 foi de 12,2 milhões de reais, distribuídos principalmente em construção de edificações comerciais, residenciais e atividades de incorporação. As experiências prévias com industrialização indicam uma concentração da utilização nos seus dois primeiros níveis e a satisfação registrada apresentou grande dispersão dentre as opiniões. Ainda que a adesão aos processos industrializados tenha se mostrado baixa, sobretudo para os níveis 3 e 4, 89\% dos respondentes acreditam que a adesão crescerá nos próximos 5 a 10 anos.

A obtenção de um canteiro mais limpo foi o benefício que obteve maior média $(4,52)$. Outros quatorze benefícios resultaram em pontuações médias iguais ou superiores a 4, atribuídos a características relacionadas ao tempo, à qualidade, aos impactos ambientais e à gestão do canteiro.
A análise dos desvios-padrões infere que há maior divergência de opiniões para as barreiras à industrialização do que para os benefícios. Este fato havia sido observado durante Workshops descritos por Blismas et al. (2005). Adicionalmente, constata-se que a barreira mais significativa - Empresas possuem pouco conhecimento/experiência - teve média de apenas 3,67, situando-se, portanto, entre as opiniões neutras (3) e a concordância parcial (4).Já 83\% das 29 barreiras levantadas tiveram médias entre 2,5 e 3,5 .

Quando solicitados a elencar os três principais motivadores, as maiores frequências ocorreram em fatores relacionados ao tempo de obra, à produtividade, à qualidade e ao custo. Os desafios apresentaram frequências mais distribuídas, sendo os principais fatores relacionados à esfera financeira e ao processo de planejamento. Os resultados desta seção corroboram o levantamento de Gibb e Isack (2003).

As percepções levantadas auxiliam na identificação dos principais benefícios percebidos pelos profissionais de construtoras e incorporadoras curitibanas sobre industrialização, além de relacionar os principais desafios e barreiras percebidos.

Para estudos futuros, sugere-se que tais percepções sejam levantadas por estados e em todo o território nacional. Baseando-se em exemplos internacionais como o IMMPREST, entende-se que a elaboração de tal base de dados servirá como auxílio para a tomada de decisão quanto aos processos construtivos, além de possibilitar a criação de ferramentas de suporte para stakeholders. 


\section{BUILDERS AND \\ DEVELOPERS' \\ PERCEPTIONS \\ ABOUT CIVIL \\ CONSTRUCTION \\ INDUSTRIALIZATION \\ IN CURITIBA}

\begin{abstract}
Although recognized for its potential to boost the expansion of the construction industry, prefabrication represents only a small portion of the current Brazilian market. This paper intends to analyze builders and developers'levelofknowledge and acceptance on the use of industrialized constructive processes for buildings in the city of Curitiba/PR, in order to contribute to the decision-making between traditional and industrial processes. Through a survey with professionals from the region, it was possible to draw a profile of the participating companies as well as their previous experiences with industrialization. In addition, the main perceptions on barriers and benefits when using prefabrication techniques were analyzed. The benefits that showed the greatest relationship with the prefabrication were attributed to the characteristics related do time, quality, environmental impacts and site management, being a reduction of time and the increase of production of the greatest motivators for companies. As for barriers, lack of knowledge / experience and longer planning periods were the items with the highest agreement regarding to the relationship with industrialization. However, the biggest challenges for implementing such techniques were: lack of prefabrication specification on design and the increase in cost. Lastly, it is suggested to continue this paper with an expansion of the scope for the entire country.
\end{abstract}

KEYWORDS: Industrialization.

Civil Construction. Benefits.

Barriers. Motivators.

\section{REFERÊNCIAS}

ASSOCIAÇÃO BRASILEIRA DE NORMAS TÉCNICAS. NBR 9062: Projeto e execução de estruturas de concreto pré-moldado. Rio de Janeiro. 2006.

AYE, L. et al. Life cycle greenhouse gas emissions and energy analysis of prefabricated reusable building modules. Energy and Buildings, v. 47, p. 159-168, 2012.

AZHAR, S.; LUKKAD, M. Y.; AHMAD, I. An investigation of critical factors and constraints for selecting modular construction over conventional stick-built technique, International Journal of Construction Education and Research, v. 9, n. 3, p. 203-225, 2013.

BABBIE, E. Métodos de pesquisa de survey. Tradução Guilherme Cezarino. Belo Horizonte: UFMG, 2003.

BARTLETT, J. E.; KOTRLIK, J. W.; HIGGINS, C. C. Organizational research: determining appropriate sample size in survey research. Information Technology, Learning, and Performance Journal, v. 19, n. 1, 2001.

BLISMAS, N. G.; PASQUIRE, C. L.; GIBB, A. G. F. IMMPREST: Interactive method for measuring pre-assembly and standardisation benefit in construction. Loughborough University, Loughborough, 2003.

BLISMAS, N. G. et al. Constraints to the use off-site production on construction projects. architectural engineering and design management. Architectural Engineering and Design Management, v. 1, n. 3, p. 153-162, 2005.

BLISMAS, N. G.; WAKEFIELD, R. Drivers, constraints and the future of offsite manufacture in Australia, Construction Innovation, v. 9 n. 1 , p.72-83, 2009.

CÂMARA BRASILEIRA DA INDÚSTRIA DA CONSTRUÇÃO (CBIC). Banco de dados: PIB Brasil e Construção Civil - Participação (\%) no valor adicionado bruto (a preços básicos) Segundo as Classes e Atividades. Disponível em: <https://goo.gl/oR2P6j>. Acesso em: 14 mar. 2016. 
$\mathrm{CAO}, \mathrm{X}$. et al. A comparative study of environmental performance between prefabricated and traditional residential buildings in China.

Journal of Cleaner Production. p. 1-13, 2015.

CENTRO DE GESTÃO E ESTUDOS
ESTRATÉGICOS (CGEE). Relatório
prospectivo setorial. Brasília: CGEE, 2009.

CONSTRUCTION INDUSTRY

DEVELOPMENT BOARD (CIDB), Manual

for IBS content scoring system (IBS Score).

Malasia: CIDB, 2010.

CONSTRUCTION INDUSTRY INSTITUTE (CII). Preliminary research on prefabrication, pre-assembly, modularization and offsite fabrication in construction. University of Texas, Austin, Texas, 2002.

EGAN, J. Rethinking construction. Department of the Environment, Transport and the Regions, Londres, 1998.

\section{FUNDAÇÃO JOÃO PINHEIRO. Déficit}

habitacional no Brasil 2013. Centro de estatística e informações (CEI). Belo Horizonte, 2015.

GIBB, A. G. F. Client's guide and tool kit for standardisation and pre-assembly, Reino Unido: CONSTRUCTION INDUSTRY RESEARCH AND INFORMATION ASSOCIATION (CIRIA), 2000..

GIBB, A. G. F.; ISACK, F. Re-engineering through pre- assembly: client expectations and drivers. Building Research and Information, v. 31, n. 2, p. 146-160. 2003.

GIL, A. C. Como elaborar projetos de pesquisa. 4. ed. São Paulo: Atlas, 2002.

GOODIER, C. I.; GIBB, A. G. F. Barriers and opportunities for osite in the UK. In: CIB JOINT INTERNATIONAL SYMPOSIUM, 2005, Helsinki, Finlândia, Proceedings...

Helsinki, Finlândia, 2005, p. 148-158.

Future opportunities for offsite in the UK. Construction Management and Economics, v. 25 , n. 6, p. 585-595, 2007.
HEWAGE K. N.; RUWANPURA, J.Y. Carpentry workers issues and efficiencies related to construction productivity in commercial construction projects in Alberta. Canadian Journal of Civil Engineering, v. 33, n. 8, p.1075-1089, 2006.

INSTITUTO BRASILEIRO DE GEOGRAFIA E ESTATÍSTICA (IBGE). Pesquisa anual da indústria da construção, v. 23. Rio de Janeiro: IBGE, 2013. Disponível em: <https://goo.gl/uVyBp6>. Acesso em: 19 abr. 2016.

JAILLON, L.; POON, C. S. Life cycle design and prefabrication in buildings: A review and case studies in Hong Kong. Automation in Construction. p. 1-8, 2013.

KOSKELA, L. An exploration towards a production theory and its application to construction. Tese de doutorado. Helsinki University of Technology, Espoo, Finlândia, 2000.

LI, Z.; SHEN, G. Q.; ALSHAWI, M. Measuring the impact of prefabrication on construction waste reduction: An empirical study in China. Resources, Conservation and Recycling, v. 91, p. 27-39, 2014.

LU, N.; LISKA, R. W. Designers' and general contractors' perceptions of offsite construction techniques in the united state construction industry. International Journal of Construction Education and Research, v. 4, n.3, p. 177-188, 2008.

LU. N. Investigation of designers' and general contractors' perceptions of offsite construction techniques in the united states construction industry. Dissertação. Clemson University, Clemson, Estados Unidos, 2007.

MAO, C.; SHEN, Q.; SHENA, L.; TANG, L.

Comparative study of greenhouse gas emissions between off-site prefabrication and conventional construction methods: Two case studies of residential projects. Energy and Buildings, v. 66, p. 165-176, 2013.

MONAHAN, J.; POWELL, J. C. An embodied carbon and energy analysis of modern methods of construction in housing: A case study using a lifecycle assessment framework. Energy and Buildings, v. 43, p. 179-188, 2010.

MONTEIRO FILHA, D. C. et al. Construção civil no Brasil: investimentos e desafios. 1. ed. Rio de Janeiro: BNDES, 2010. 


\section{SOBRE O AUTOR}

O'CONNOR, J. T.; O'BRIEN, W. J.; CHOI, J. O. Critical success factors and enablers for optimum and maximum industrial modularization. Journal of Construction Engineering and Management (ASCE), v. 140, n. 6, p. 04014012-1-04014012-11, 2014.

OCHOA, C. Calculadora de amostra para proporções. 2013. Disponível em: <https://goo.gl/ JfTKzT >. Acesso em: 12/06/2016.

Qual é o tamanho da amostra que eu preciso? 2013. Disponível em: <https://goo.gl/ WZS4XL >. Acesso em: 12/06/2016.

PAN, W.; GIBB, A. G. F.; DAINTY, A. R. J.; Perspectives of UK housebuilders on the use of offsite modern methods of construction. Construction Management and Economics, v. 25, n. 2, p. 183-194. 2007.

PENDLEBURY, M. C.; GIBB, A. G. F. Standardisation and pre-assembly - capturing Clients requirements. In: CLIENTS DRIVING INNOVATION CONFERENCE, 2004, Queensland, Proceedings... Queensland, 2004, p. 1-11.

RAHMAN, M. M.; Barriers of implementing modern methods of construction. Journal Of Management In Engineering, v. 30, n. 1, p. 69-77. 2014.

\section{SINDICATO DA INDÚSTRIA DA CONSTRUCAO CIVIL - SINDUSCON. FUNDAÇÃO GETULIO VARGAS. \\ Produtividade na construção. 2015.36 p.}

TAM, V.W. Y. et al. Towards adoption of prefabrication in construction. Building and Environment, v. 42, p. 3642-3654, 2007.

WANG, J.; LI, Z.; TAM, V. W. Y. Identifying best design strategies for construction waste minimization. Journal of Cleaner Production. p. 1-11, 2015.

\section{WASTE \& RESOURSES ACTION} PROGRAMME - WRAP. Current practices and future potential in modern methods of construction. Banbury, Reino Unido, 2007.

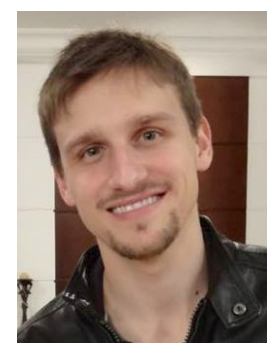

\section{Eduardo Werneck}

Senger

Graduado em engenharia civil pela Universidade Federal do Paraná (UFPR), cursou parte da graduação no Karlsruher Institut für Technologie (KIT), na Alemanha.Concluiu um MBA em Gestão de Obras de Edificações no SENAI e atualmente é mestrando do Programa de Pós-Graduação em Engenharia de Construção Civil (PPGECC) da UFPR, com tema de dissertação em análise de valor de banheiros tradicionais para estabelecimento de comparativo com banheiros industrializados.Possui experiência na área de Engenharia Civil, com ênfase em Processos Construtivos e tem interesse em sistemas construtivos inovadores que contribuam com os três pilares da sustentabilidade na construção civil: ambiental, social e econômico.Lecionou engenhariacivil na Faculdade Educacional de Araucária e tem se dedicado aos processos construtivos industrializados, especificamente aos banheiros pré-fabricados.

\section{****}

Data de recebimento: 20/03/2017

Data de aprovação: 24/08/2017 